\title{
PROFESIONALIZACIÓN DE LA GESTIÓN DESDE EL PROGRAMA GAUCHO DE CALIDAD Y PRODUCTIVIDAD: EL CASO DE UNILASALLE DE BRASIL
}

\author{
Paulo Fossatti ${ }^{1}$, Renaldo Vieira de Souza ${ }^{2}$ y \\ Francisco Ganga ${ }^{3}$
}

\begin{abstract}
RESUMEN
Es innegable que en los últimos años se está asistiendo a un reposicionamiento de la importancia que tiene la formación de capital humano avanzado y la gestión estratégica en las organizaciones. A partir de esta realidad, este trabajo se ha planteado como objetivo central analizar los resultados del Programa Gaucho de Calidad y Productividad (PGQP) en la profesionalización de la gestión del Unilasalle Canoas, Brasil. Se trata de un estudio de caso, cualitativo, con análisis documental que comprendió: Plan de Desarrollo Institucional (PDI); Informe de Gestión sobre el Programa Gaucho de Calidad y Productividad -RG-PGQP 2013 (por sus siglas en portugués)- e Informe Balance Scorecard (BSC, por sus siglas en inglés) 2010-2013. Los resultados se traducen en discursos y prácticas emergentes para los criterios del PGQP que colaboran en la profesionalización del modelo de gestión universitaria de Unilasalle: liderazgo, estrategias y planes, cliente, sociedad, información y conocimiento, personas, procesos y resultados. Los resultados sugieren la consolidación de los procesos de gestión de la institución a partir de la respectiva herramienta en análisis.
\end{abstract}

Palabras clave: universidades, calidad, gestión de la educación superior, modelo de gestión, Programa Gaucho de Calidad y Productividad.

\section{THE PROFESSIONALIZATION OF MANAGEMENT FROM THE GAUCHO QUALITY AND PRODUCTIVITY PROGRAM: THE CASE OF UNILASALLE BRAZIL}

\section{ABSTRACT}

It is undeniable that over the past few years we have witnessed a repositioning of the importance of the development of human capital and advanced strategic management in organizations. Given this reality, this study's central objective is to analyze the results of the Gaucho Program of Quality and Productivity (PGQP) in the professionalization of the management of the University, Unilasalle Canoas, Brazil. It is a qualitative case study, with a documentary analysis of: Institutional Development Plan (IDP); Management Report on the Gaucho Program of Quality and Productivity -RG-PGQP 2013 (by its Portuguese acronym)- and the Report Balanced Scorecard (BSC) 2010-2013. The results are translated into speeches and emerging practices for the criteria of the PGQP that contribute to the professionalization of the university management model of Unilasalle: leadership, strategies and plans, client, society, information and knowledge, people, processes and results. The results suggest the consolidation of the management processes of the institution from the respective tool in analysis.

Key Words: universities, quality, higher education management, management model, Gaucho Quality and Productivity Program.

1 Centro Universitario La Salle de Canoas, Rio Grande do Sul, Canoas, Brasil. Correo electrónico: irpaulo@unilasalle.edu.br

2 Centro Universitario La Salle de Canoas, Rio Grande do Sul, Canoas, Brasil. Correo electrónico: renaldo.souza@unilasalle.edu.br

3 Departamento de Ciencias del Desarrollo de la Universidad de Los Lagos, Campus Santiago, Santiago, Chile. Correo electrónico: fganga@ulagos.cl 
162 PROFESIONALIZACIÓN DE LA GESTIÓN DESDE EL PROGRAMA GAUCHO DE CALIDAD Y PRODUCTIVIDAD: EL CASO DE UNILASALLE DE BRASIL - P. Fossatti, R. Vieira de Souza y F. Ganga

\section{Introducción}

La evolución de la concepción de calidad retratada en las últimas décadas -como los modelos de excelencia en gestión, identificados por los premios de calidad- se presenta como opción relevante en la perspectiva de lograr mejoras en el desempeño de las organizaciones. Los principales propósitos de estos modelos de excelencia en gestión son: difundir y promover la concientización para la calidad y estimularla en la mejoría de la excelencia y competitividad, en los diversos tipos de organizaciones de las diferentes partes del mundo (Cauchick Miguel, 2004).

La profesionalización de la gestión es una realidad en las Instituciones de Enseñanza Superior (IES) y debe de ser cada vez más difundida frente a la necesidad de preparación de estas entidades, haciéndolas más competitivas.

En el seguimiento de servicios, en especial de la educación superior, se percibe un expresivo aumento de instituciones privadas, consiguiendo un espacio significativo en la economía global. Sin embargo, este incremento aún carece de una gestión más profesionalizada en las organizaciones tradicionales, frente al crecimiento de nuevas instituciones que ya surgen fuertes y en cadena, provocando el aumento en la competitividad, el abigarramiento contextual y la necesidad de mayores niveles de eficiencia organizacional (Ganga y Burotto, 2012, Ganga et al., 2014). En este orden de cosas, la complejidad del mundo moderno -que gesta una nueva mirada institucional-requiere innovadoras posturas en relación con la gestión, como apunta Drucker (2009):

Hace veinte años, administración era un nombre no hermoso para los involucrados en las organizaciones sin fines lucrativos. Significaba negocios, y esas entidades se enorgullecían de estar libres de la falla del comercialismo y arriba de preocupaciones sórdidas, como la línea de resultados. Actualmente, gran parte de ellas ya comprendió que las entidades sin fines lucrativos necesitan administración hasta más que las empresas, exactamente porque les falta la disciplina impuesta por la línea de resultados. Es evidente que esas entidades siguen dedicadas a "hacerlo bien", pero también ya comprendieron que 
buenas intenciones no sustituyen organización y liderazgo, responsabilidad, desempeño y resultados (p. 63).

Estudios apuntan a la existencia de aproximadamente ochenta países que utilizan modelos de excelencia de gestión en el nivel nacional, como es el caso de Brasil (Cauchick Miguel, 2004). Los precursores de los prototipos existentes fueron desarrollados en Australia, Canadá, Estados Unidos y Japón. Hoy aún tienen un gran impacto mundial el Malcolm Baldrige National Quality Award (MBNQA) de Estados Unidos y el modelo regional representado por el European Quality Award (EQA).

Diversos modelos fueron desarrollados en Europa en los últimos quince años, pero el EQA, administrado por la European Foundation for Quality Management, congrega a más de 750 organizaciones europeas (Cauchick Miguel, 2004). A su vez, el MBNQA es la base para diversos modelos en el mundo, entre ellos el Premio Nacional de Calidad (PNQ, por sus siglas en portugués) de Brasil, que es comparable a diversos galardones importantes a nivel global (Cauchick Miguel, 2004).

Según datos históricos extraídos de la página web del Movimiento Brasil Competitivo (MBC, 2012) y la Fundación Nacional de Calidad (FNQ, 2011):

El PNQ fue establecido hace dos décadas como una importante herramienta de estructuración de un modelo de gestión y de mejoría de la calidad, productividad y desempeño de organizaciones instaladas en el país. Desde entonces, entidades de diversos sectores económicos organizan sus prácticas de gestión de acuerdo con los criterios del PNQ. Diversas empresas de los sectores industriales y de prestación de servicios alcanzan éxito, no solamente en cuestión de resultados organizacionales, sino también recibiendo el reconocimiento nacional representado por la premiación (p. 11).

En el marco de ese período surge el Programa Gaucho de Calidad y Productividad (PGQP, por sus siglas en portugués) que se consolida contribuyendo a que el Estado de Rio Grande do Sul tenga una fuerte influencia en la creación del Movimiento Brasil Competitivo (MBC). Así, queda clara la intención de dicho programa 
de no contribuir solamente en su Estado, sino también ayudar a llevar los conceptos de calidad a todo el país.

Nacionalmente, el PGQP apoya el fortalecimiento y ampliación de la cadena nacional de calidad, productividad y competitividad, gracias a la sociedad entre MBC y FNQ (MBC, 2012).

En relación con la definición de la nueva modalidad y reglamentación de la institución estudiada, esta se basa en la Ley $12.881 / 2013$ que especifica claramente a las Instituciones Comunitarias de Educación Superior (ICES), como aquellas IES de carácter privado sin fines lucrativos.

En este contexto, el artículo tiene como finalidad cardinal, analizar los resultados del Programa Gaucho de Calidad y Productividad en la profesionalización de la gestión de la Universidad de La Salle de Canoas, Brasil.

Desde el punto de vista metodológico, se puede decir que se trata de un estudio de caso, tipo cualitativo, con análisis documental. Los resultados se traducen en discursos y prácticas emergentes de los criterios del PGQP que colaboran en la profesionalización del modelo de gestión universitaria del Unilasalle. Se sugiere la consolidación de los procesos de gestión de la institución tomando en consideración la herramienta en análisis. En este sentido, la pregunta de investigación busca identificar hipotéticamente si la adopción de modelos y prácticas de gestión contribuye a una gestión profesional y puede reflejar resultados significativos para el desarrollo institucional.

En cuanto a la estructuración del contenido, en primera instancia se presenta el marco teórico; para luego dar paso a los aspectos metodológicos y la correspondiente discusión de los resultados; concluyendo con las respectivas consideraciones finales. 


\section{Marco teórico}

\subsection{Aspectos legales para la institución comunitaria y filantrópica}

El modelo de gestión en análisis está basado en el estudio de una institución confesional, comunitaria y filantrópica que tiene como base la Ley 12.881 de 12 de noviembre de 2013 (Presidência da República, 2013) y que define a las instituciones comunitarias de educación superior como:

Art. 1ํ.- Las Instituciones Comunitarias de Educación Superior son organizaciones de la sociedad civil brasileña que poseen, acumulativamente, las siguientes características:

I. Están constituidas en la forma de asociación o fundación, con personalidad jurídica de derecho privado, inclusive las instituidas por el poder público.

II. Patrimonio que pertenece a las entidades de la sociedad civil y/o poder público.

III. Sin fines lucrativos, así comprendidas, las que observan, acumulativamente, los siguientes requisitos:

(...) Las Instituciones Comunitarias de Educación Superior institucionalizarán programas permanentes de extensión y acción comunitaria, direccionadas a la formación y desarrollo de los alumnos y de la sociedad (Párrafo $4^{\circ}$ ).

\subsection{Universidades lasallistas}

Las IES en estudio pertenecen a la International Association of Lasallian Universities (IALU), y poseen siglos de tradición en educación en todas las áreas del conocimiento. Actualmente existen 75 IES lasallistas alrededor del mundo (IALU, 2013).

La educación lasallista está anclada en un concepto de educación que reúne "el todo de la persona" y, al mismo tiempo, todo el transcurrir de la vida. Esa comprensión incluye y transciende la lógica de la educación escolar formal. Busca garantizar -conjuntamente con las habilidades y las competencias propias de cada etapa formativaideales y valores responsables de una formación humana y cristiana de calidad, que dé unidad y sentido a la vida (Fossatti, Hengemule, $\&$ Casagrande, 2011). 
El Centro Universitario La Salle, Unilasalle, está ubicado en una de las regiones más prósperas y productivas de Brasil, la ciudad de Canoas, Estado de Rio Grande do Sul. Tiene su historia conectada con la trayectoria de las obras educativas lasallistas, las cuales se originaron en la propuesta educativa de Juan Bautista de La Salle (1651-1719), quien se dedicó a la creación de escuelas para niños de clases menos favorecidas y fundó la institución educativa lasallista, cuyo objetivo central es la dedicación a la educación de niños, jóvenes y adultos y a la formación de profesores.

Desde Francia, la actuación de los lasallistas se ha difundido por el mundo. Actualmente están presentes en más de 80 países y cuentan con aproximadamente 60.000 educadores y alrededor de 1.500 instituciones educativas que acogen a aproximadamente un millón de niños, jóvenes y adultos.

\subsection{La gestión estratégica universitaria}

Según afirman Braga y Monteiro (2005), gestión es un proceso pragmático en el que interesa el resultado y no el esfuerzo. Dicha afirmación refleja la forma como se mira la gestión desde el punto de vista estratégico, donde existe la necesidad de una complicidad mayor con los resultados más que con los desafíos de administrar la institución como un negocio que tenga retorno; también implica garantizar la sustentabilidad operacional y financiera.

De esta manera, es necesario desarrollar el aprendizaje continuo de las personas que lideran y son responsables por las decisiones, encarando la gestión como ciencia, buscando el entendimiento de las circunstancias y la adopción de acciones reflejadas en esos escenarios. Siguiendo con Braga y Monteiro (2005), gestión es también la búsqueda de criterios para la toma de decisiones, basada en evidencia empírica y en su valor predicho.

De acuerdo con Betancourt (2002), Cortadellas (2007), Bustos, Zapata y Ramírez (2008), Llinàs-Audet y Solé (2011) y Ganga et al. (2015), para garantizar que las decisiones sean las más asertivas posibles, tenemos que considerar modelos orientadores y también la relación existente entre la gestión y el proceso decisional. Esta 
postura ayudará a la organización a caminar rumbo a los propósitos previamente definidos.

Aún en el ámbito educativo, De la Orden et al. sostenían en el año 1997, (ratificado el año 2012 por el mismo De la Orden), que lo que se llamaba genéricamente "calidad de la educación" era un complejo constructo valorativo apoyado en la consideración conjunta de tres dimensiones interrelacionadas: funcionalidad, eficacia y eficiencia; expresión de un sistema de coherencias múltiples entre los componentes básicos de los sistemas y centros educativos. De modo más sintético, Rodríguez (1991) ha expresado que la calidad de la universidad radica en su capacidad para alcanzar el mayor y mejor desarrollo factible de cada uno de sus integrantes.

Finalmente, Yañez (2012) expresa que la educación de calidad es aquella que permite el desarrollo social, la que educa a hombres y mujeres luchadores por un mundo mejor, por la plenitud de todas sus capacidades y talentos y por el desarrollo íntegro de una sociedad humanista constituida por todos y no solo por los que poseen los recursos económicos para la llamada "vida digna".

\subsection{El trayecto del MBC y del PGQP}

La búsqueda de la excelencia en gestión siempre ha tenido foco en los resultados, esto es, en la mejoría de los procesos, productos y todos los elementos que componen una organización. Pero una buena metodología de calidad posibilita su aplicación en cualquier organización, de todo tamaño y sector.

De la página web del MBC -donde el PGQP está inserto- se extrajeron los siguientes comentarios:

¿Cómo el PGQP podría encontrar una metodología que pudiera ser aplicada desde una microempresa hasta una organización con millones de funcionarios? La respuesta de eso fue el Modelo de Excelencia en Gestión (MEG), una herramienta completa que tiene todos los requisitos necesarios para que se haga una buena gestión. Está basada en once fundamentos: pensamiento sistemático, aprendizaje organizacional, cultura de innovación, liderazgo y constancia de propósitos, 
orientación por procesos e informaciones, visión de futuro, generación de valor, valoración de personas, conocimiento sobre el cliente y el mercado, desarrollo de sociedades y responsabilidad social. Y ocho criterios: liderazgo, estrategias y planes, clientes, sociedad, información y conocimiento, personas, procesos y resultados. Esta herramienta fue creada por la Fundación Nacional de Calidad (FNQ, 2011).

El esquema representativo del MEG simboliza la organización, considerada como un sistema orgánico y adaptable al medio externo. El MEG es representado por la Figura 1, que utiliza el concepto de aprendizaje según el ciclo del Plan, Do, Check, Action (PDCA) (MBC, 2012).

Figura 1: Diagrama del Modelo de Excelencia de la Gestión (MEG)

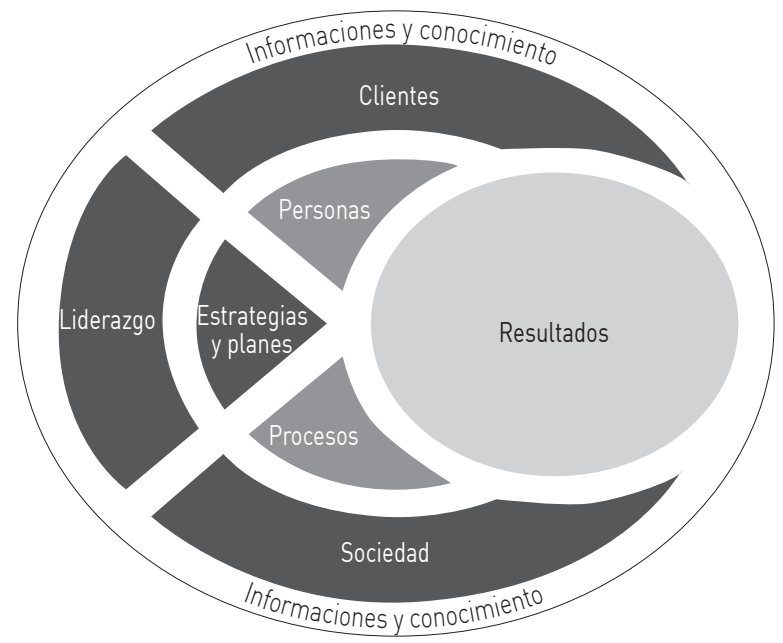

Fuente: FNC (2011).

En general existe cierto consenso en señalar que el éxito de una organización está directamente relacionado con su capacidad para satisfacer las necesidades y expectativas de sus clientes. Ellas deben ser identificadas, comprendidas y utilizadas para que sea creado el valor necesario, que posibilite conquistar y mantener esos clientes.

Por su parte Zúñiga (2007), citando las normas ISO, indica que la calidad puede ser definida como el grado en que un conjunto de características inherentes cumple con unos requisitos. 
Para que haya continuidad en sus operaciones, las organizaciones deben también identificar, comprender y satisfacer las necesidades y expectativas de la sociedad y de las comunidades con las cuales hay interacción, siempre de manera ética, cumpliendo las leyes y preservando el ambiente (MBC, 2012).

La metodología que exalta el PGQP especifica los detalles y acomodos necesarios para la adhesión al modelo, de manera que se oriente a las personas en los diferentes niveles de la organización y, a partir de esta guía, se desplieguen las fases necesarias para confrontar las prácticas administrativas de la organización con el modelo de gestión.

Esas informaciones representan la inteligencia de la organización, viabilizando el análisis del desempeño y ejecución de las acciones necesarias en todos los niveles. La gestión de la información y de los activos inasequibles es un elemento esencial en la búsqueda de la excelencia. El MEG está basado en once fundamentos y ocho criterios con los cuales podemos definir la base teórica de una buena gestión.

\subsection{Los once fundamentos}

En la página web del MBC (2012) se describen los valores organizacionales que pueden ser percibidos como parte de la cultura de las instituciones y que deben ser practicados desde los líderes de mayor nivel hasta los empleados menores. Estas prácticas son la base para la formación de una cultura de gestión enfocada en los resultados y darán origen a los criterios de evaluación (ver Tabla 1). 

CALIDAD Y PRODUCTIVIDAD: EL CASO DE UNILASALLE DE BRASIL - P. Fossatti, R. Vieira de Souza y F. Ganga

Tabla 1: Valores organizacionales

\begin{tabular}{|c|c|}
\hline $\begin{array}{l}\text { Valores } \\
\text { organizacionales }\end{array}$ & Definición \\
\hline $\begin{array}{l}\text { Pensamiento } \\
\text { sistémico }\end{array}$ & $\begin{array}{l}\text { Entendimiento de las relaciones de interdependencia entre los diversos } \\
\text { componentes de una organización, así como entre la organización y el } \\
\text { ambiente externo. }\end{array}$ \\
\hline $\begin{array}{l}\text { Aprendizaje } \\
\text { organizacional }\end{array}$ & $\begin{array}{l}\text { Búsqueda y extensión de un nuevo rellano de conocimiento para la } \\
\text { organización por medio de la percepción, reflexión, evaluación y compartir } \\
\text { experiencias. }\end{array}$ \\
\hline $\begin{array}{l}\text { Cultura de } \\
\text { innovación }\end{array}$ & $\begin{array}{l}\text { Promoción de un ambiente favorable para la creatividad, experimentación } \\
\text { e implementación de nuevas ideas que puedan generar un diferencial } \\
\text { competitivo para la organización. }\end{array}$ \\
\hline $\begin{array}{l}\text { Liderazgo y } \\
\text { constancia de } \\
\text { propósitos }\end{array}$ & $\begin{array}{l}\text { Actuación de manera abierta, democrática, inspiradora y motivadora de las } \\
\text { personas, buscando el desarrollo de la cultura por excelencia, la promoción } \\
\text { de relaciones de calidad y la protección de los intereses de las partes } \\
\text { interesadas. }\end{array}$ \\
\hline $\begin{array}{l}\text { Orientación hacia } \\
\text { los procesos e } \\
\text { informaciones }\end{array}$ & $\begin{array}{l}\text { Comprensión y segmentación del conjunto de las actividades y procesos de } \\
\text { la organización que añade valor a las partes interesadas, siendo que la toma } \\
\text { de decisiones y ejecución de acciones debe tener como base la medición } \\
\text { y análisis del desempeño, teniendo en consideración las informaciones } \\
\text { disponibles, además de incluir los riesgos identificados. }\end{array}$ \\
\hline Visión de futuro & $\begin{array}{l}\text { Comprensión de los factores que afectan la organización, su ecosistema y el } \\
\text { ambiente externo en corto o largo plazo. }\end{array}$ \\
\hline $\begin{array}{l}\text { Generación de } \\
\text { valor }\end{array}$ & $\begin{array}{l}\text { Búsqueda de resultados consistentes con el aumento de valor tangible e } \\
\text { inalcanzable de manera sostenida para todas las partes interesadas. }\end{array}$ \\
\hline $\begin{array}{l}\text { Valoración de las } \\
\text { personas }\end{array}$ & $\begin{array}{l}\text { Creación de condiciones para que las personas se realicen profesional y } \\
\text { humanamente, maximizando su desempeño por medio del compromiso, } \\
\text { desarrollo de competencias y espacios para emprender. }\end{array}$ \\
\hline $\begin{array}{l}\text { Conocimiento } \\
\text { del cliente y el } \\
\text { mercado }\end{array}$ & $\begin{array}{l}\text { Conocimiento y entendimiento del cliente y del mercado, visando la } \\
\text { creación de valor de manera sostenida para el cliente y, consecuentemente, } \\
\text { generando mayor competitividad en los mercados. }\end{array}$ \\
\hline $\begin{array}{l}\text { Desarrollo de } \\
\text { sociedades }\end{array}$ & $\begin{array}{l}\text { Desarrollo de actividades en conjunto con otras organizaciones a partir de } \\
\text { la total utilización de las competencias esenciales de cada una, objetivando } \\
\text { beneficios para todas las partes. }\end{array}$ \\
\hline $\begin{array}{l}\text { Responsabilidad } \\
\text { social }\end{array}$ & $\begin{array}{l}\text { Actuación que se define por la relación ética y transparente de la } \\
\text { organización con todo el público con el cual se relaciona. Se refiere también } \\
\text { a la inserción de la empresa en el desarrollo sustentable de la sociedad, } \\
\text { preservando recursos ambientales y culturales para las generaciones } \\
\text { futuras, respetando la diversidad y promoviendo la reducción de las } \\
\text { desigualdades sociales como parte integrante de la estrategia de la } \\
\text { organización. }\end{array}$ \\
\hline
\end{tabular}

Fuente: MBC (2014). Adaptada por los autores, basado en Senge (1992). 


\subsection{Los ocho criterios de excelencia}

Conforme a la FNQ (2011), los criterios de excelencia están dibujados no solamente para servir de modelo de evaluación para el PGQP, sino también -y de manera principal- para ser utilizados como diagnóstico del sistema de gestión de cualquier tipo de organización. Los ocho criterios de excelencia se refieren a: liderazgo, estrategias y planes, clientes, sociedad, información y conocimiento, personas, procesos y resultados.

En consecuencia, de la estructura antes puntualizada, los ocho criterios de excelencia se subdividen en 24 elementos establecidos por una convención como "ítems de procesos gerenciales" que describen de manera específica algunos requisitos relacionados con la gestión de la organización, sin prescribir herramientas o métodos de trabajo específicos. Los ítems de resultados organizacionales requieren de la presentación de indicadores que muestren el desempeño en áreas de resultados importantes.

\section{Aspectos metodológicos}

La investigación, de la cual proviene esta producción, se caracteriza por ser un estudio de caso acerca del modelo de gestión de una IES en el sur de Brasil, donde el abordaje cuantitativo ayuda al enfoque cualitativo. El objetivo general de la investigación es analizar cómo el modelo de gestión adoptado por las IES contribuye al desarrollo sistémico de los ejes: identidad de la IES, herramientas de gestión, aprendizaje y crecimiento de la IES, sustentabilidad (económica y financiera, social y ambiental) y calidad de vida en la organización.

Para Fox (1981), luego de la decisión del investigador respecto del problema investigativo y, en consonancia con este, del marco teórico que sustentará el desarrollo del estudio, se torna necesario definir el universo pertinente a la investigación, el cual corresponde a la Unilasalle Canoas. Esta opción ocurre por el proceso de transformación de la gestión vivida desde 2006, que arrojó señales de relevantes resultados y también debido a la relación que los investigadores establecen con la misma. 
172 PROFESIONALIZACIÓN DE LA GESTIÓN DESDE EL PROGRAMA GAUCHO DE CALIDAD Y PRODUCTIVIDAD: EL CASO DE UNILASALLE DE BRASIL - P. Fossatti, R. Vieira de Souza y F. Ganga

Sin embargo, este artículo es un extracto de la investigación arriba referida, donde se intenta abordar la contribución de la herramienta de gestión PGQP, para la profesionalización de la gestión del Unilasalle Canoas. Por lo tanto, en este documento se analizan los resultados del PGQP y sus efectos en la profesionalización de la gestión de la institución universitaria antes citada.

Para llegar a los resultados previamente proyectados, se ha realizado un análisis documental que comprendió: Plan de Desarrollo Institucional (PDI), PGQP e Informe Balance Scorecard (BSC) 20102013 del Centro Universitario La Salle (2013).

Tal análisis se apoya, aunque no se limita, en la propuesta de análisis de contenido ofrecida por Bardin (2011) para examinar el contenido emergente de los siete criterios propuestos por la PGQP. Según la autora, las diferentes fases del análisis de contenido son: el preanálisis, la exploración de la materia, el tratamiento de los resultados y la inferencia e interpretación de los resultados.

Considerando que metodológicamente se comenzó con siete criterios predefinidos por el PGQP, también se presenta un sustento en Bardin (2011) para el análisis del contenido emergente de cada criterio.

En relación con la formulación de la problemática, se puede indicar que la interrogante investigativa tenía como finalidad central identificar hipotéticamente si la adopción de modelos y prácticas de gestión contribuyen concluyentemente a una gestión profesional y que ello se refleja en productos relevantes para el desarrollo institucional.

\section{Presentación de los resultados}

Para identificar los resultados fue necesario efectuar un estudio que permitiera la definición de los indicadores correspondientes en el plan estratégico. Este proceso se hizo a partir de un enfoque que involucró a los máximos directivos a través de una serie de reflexiones y análisis, asentados en una mirada sistémica fundamental, lo que permitió identificar la necesidad de los elementos básicos de un plan 
estratégico (misión, visión, principios y valores), para elaborar el mapa estratégico correspondiente, como se muestra en la Figura 2.

Figura 2: Diagrama del Modelo de Excelencia de la Gestión (MEG)

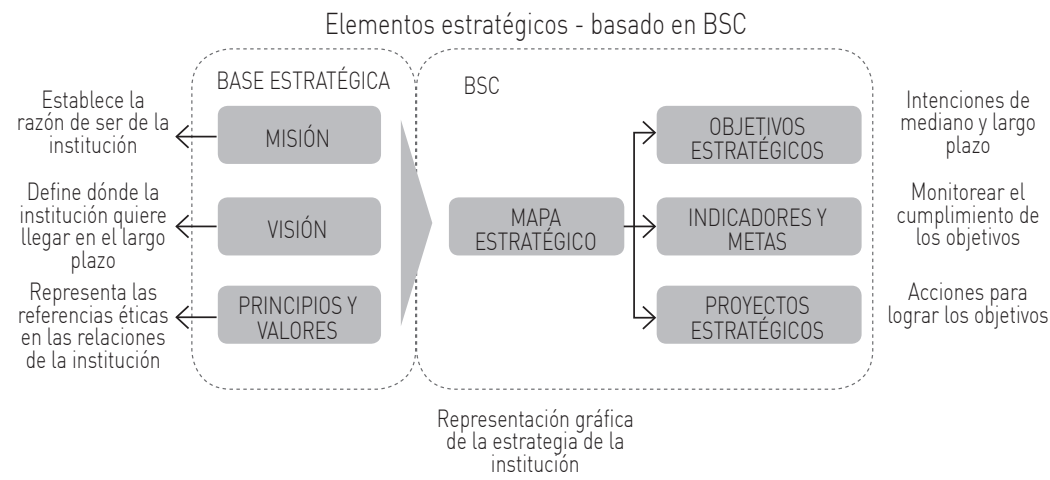

Fuente: Centro Universitario La Salle (2013).

Considerando el análisis documental -especialmente los datos del Informe de gestión sobre el PGQP (Centro Universitario La Salle, 2013) - se presentan las categorías utilizadas por la investigación, las cuales son analizadas bajo los criterios de FNQ.

\subsection{Liderazgo}

Según criterios comprometidos con la excelencia y que orientan la evaluación y diagnóstico de gestión organizacional (FNQ, 2011), el factor "liderazgo" comprende los procesos gerenciales relativos a la orientación filosófica de la organización, así como la contratación, por parte de los líderes, de las personas y partes interesadas en su causa, y el control de resultados ejercidos por la dirección.

Tomando en cuenta este criterio, la institución trata los valores y principios organizacionales que son establecidos a partir del PDI, instrumento de planificación y gestión que, además de comprender a toda la institución, considera su identidad y asegura la efectividad de sus contenidos y procesos. Por su importancia como documento que guía las acciones del Unilasalle, el PDI está disponible para los niveles institucionales, la comunidad académica y las partes interesadas. Es 
importante destacar el mayor desarrollo y participación en diferentes niveles que contribuyeron en la actualización del PDI para el ciclo 2014-2018. Los valores y principios están bajo la responsabilidad de la Rectoría, que los somete a la apreciación del Órgano Colegiado de Administración Superior (Consejo Universitario), ordenado por representantes del liderazgo, docentes, y comunidad.

Otros medios analizados que contemplan la toma de decisión son: el Programa de Evaluación Institucional (PROAVI, por sus siglas en portugués), la investigación de mercado, el Servicio de Sugerencias y Quejas, el BSC y la Evaluación del Ministerio de Educación (MEC). Así mismo, siempre se buscó un mayor involucramiento de los líderes en el proceso decisional para que luego las decisiones fueran dadas a conocer a través de diferentes dispositivos de comunicación interna, donde también son divulgados otros acontecimientos de interés de todos los colaboradores de Unilasalle.

La implementación de la herramienta BCI, así como el acompañamiento mensual de los indicadores sirve para realizar el análisis de desempeño, operacional y estratégico, considerando los indicadores del mapa estratégico vinculados con las partes interesadas. Como apoyo para dicho acompañamiento, el método utilizado para el control de la práctica y de los informes gerenciales es el software Strategic Adviser (SA).

En lo que se refiere al desarrollo de nuevos líderes, la institución utiliza el Programa de Gestión por Competencia, que ha buscado recientemente nuevas metodologías de análisis del perfil de comportamiento como el sistema DISC, que comprende cuatro factores de comportamiento: dominancia, influencia, estabilidad y conformidad.

\subsection{Estrategias y planes}

Según los criterios de compromiso con la excelencia (FNQ, 2011), el criterio "estrategias y planes" aborda los procesos gerenciales relativos a la concepción y ejecución de las estrategias, incluyendo aquellos referidos a los establecimientos de metas y a la definición y acompañamiento de los planes necesarios para el éxito de las 
estrategias. Para atender a este tópico, la Rectoría del Unilasalle, analizando la adecuación de la institución a nuevos tiempos y buscando calificar la gestión universitaria, adoptó la herramienta de gestión estratégica BSC. Con su adopción se contribuye a la definición de las estrategias de la institución y se ayuda al grupo directivo en el desarrollo del pensamiento estratégico.

En este sentido, el BSC sirvió para dibujar el mapa estratégico del Unilasalle a partir de las cuatro perspectivas favorecidas en esta herramienta: clientes, financiera, procesos internos y aprendizaje, y crecimiento, las cuales deben estar alineadas con la misión y visión institucional. Como se observa, nuevas maneras de gestión fueron adoptadas, como por ejemplo, el presupuesto base en el cual los recursos son dispuestos por rúbricas.

Es importante destacar que para el acompañamiento de los planes de acción y sus respectivos indicadores -los cuales son realizados mensualmente, en el encuentro denominado "Reunión para acompañamiento del BSC"- en el cual participan el rector, el vicerrector, los prorrectores y los directores; como también, los responsables de cada indicador y sus respectivos monitores (miembros de la Rectoría).

\subsection{Clientes}

Según los criterios de compromiso con la excelencia (FNQ, 2011), el factor "clientes" debe describir:

- Cómo la organización identifica, analiza y comprende las necesidades y expectativas de sus usuarios y de los respectivos mercados.

- Cómo divulga sus productos, marcas y acciones de mejoría.

- Cómo estrecha su relación con los clientes.

Este criterio aborda también los procesos gerenciales relativos al tratamiento de informaciones de clientes y a la comunicación con clientes actuales y potenciales.

Buscando desarrollar estudios que contribuyesen a la identificación y análisis de las necesidades y expectativas de los 
usuarios fue creado un núcleo de marketing analítico, dependiente de la Dirección de Marketing, el cual tiene como objetivo obtener y gestionar informaciones del mercado que tienen relevancia estratégica para la institución. La comunidad externa puede enviar sus opiniones a través del formulario Fale Conosco (Contáctenos), disponible en la página web del Unilasalle.

Otra acción significativa es el PROAVI. Este fue instituido para crear anualmente una base de datos de carácter evaluativo que ayudase a la institución en la búsqueda de la excelencia académica, calificación pedagógica, eficiencia administrativa y sustentabilidad financiera. Así también aspira a consolidar procesos de acompañamiento regular y sistemático de preservación, actualización y mejora de su misión educativa.

\subsection{Sociedad}

Según los criterios de compromiso con la excelencia (FNQ, 2011), el criterio "sociedad" sirve para definir cómo la organización contribuye al desarrollo económico, social y ambiental de manera sustentable. Aborda incluso los procesos gerenciales relativos al respeto y tratamiento de las demandas de la sociedad, medioambiente y desarrollo social.

Atendiendo a este criterio la Unilasalle en su constitución histórica está atenta a las dimensiones sociales y ambientales, a pesar de que sus procesos, productos y servicios generen relativamente pocos impactos. Para ello, busca la identificación y el tratamiento de los mismos, alineado con el principio del ejercicio de ciudadanía fraterna y solidaria, como el objetivo de integrarse a la comunidad local. Esta actitud pretende contribuir al desarrollo social, cultural y en la mejora de la calidad de vida, considerando sus múltiples manifestaciones.

En relación con los aspectos sociales e impactos ambientales, es importante destacar la creación del Comité de Sustentabilidad, formado por un grupo de profesores y funcionarios de varios sectores de la Unilasalle, por lo que tiene una característica multidisciplinar. En aspectos de seguridad de las personas que trabajan en la institución, 
fue establecido el programa de Prevención de Riesgos Ambientales (PPRA), para formalizar la prevención e identificación de riesgos ambientales, preservando la salud y la integridad de los colaboradores.

En la perspectiva de proyectos sociales, además de las recomendaciones de la Política Nacional de Asistencia Social (PNAS), el constante análisis de la realidad local por parte del servicio social definen la actuación y las líneas de acción social de la institución, entre las que cabe destacar el Núcleo de Práctica Jurídica, que sirve gratuitamente a la comunidad menos favorecida en causas civiles y laborales, complementando la graduación de los académicos en Derecho.

También se puede citar el Centro Salud Escuela Unilasalle, que ofrece atención médica a la población menos favorecida, actuando de manera preventiva por medio de programas integrados e involucrando a académicos y docentes del área de la salud, principalmente de los cursos de Fisioterapia, Nutrición, Enfermería y Educación Física.

La gestión de las políticas y procesos de interacción universidad, empresa y gobierno, es desarrollado en la institución por medio de un centro tecnológico y social, el Tecno Social, el cual está enfocado en la economía solidaria.

\subsection{Informaciones y conocimiento}

Según los estándares de compromiso con la excelencia (FNQ, 2011), el criterio "información y conocimiento" sirve para examinar la gestión y la utilización de la información de la organización y la gestión de sus activos intangibles. Aborda, por lo tanto, los procesos gerenciales relativos al tratamiento organizado de la demanda por información en la organización y comparte y retiene el conocimiento generador de diferenciales competitivos.

Así, son varias las acciones de mejora identificadas e implementadas en la consolidación del proceso de definición de los sistemas de información a partir de un nuevo sistema de gestión académica, con el fin de integrar los procesos de Unilasalle con la gestión de toda la cadena organizativa, garantizando la igualdad de los procedimientos entre todas las unidades. 
Con la creación del sistema Unilasalle Comparte, se trabaja la información de los niveles estratégicos, tácticos y operacionales, la cual es analizada y debatida en las reuniones de los líderes y luego es compartida y analizada con el resto de la fuerza laboral.

En relación al know how en la enseñanza superior, el factor diferenciador de la Unilasalle está en promover una educación que coloca en primer lugar el lado humano, siendo fiel a los principios lasallistas. Por ello, se recibe a los académicos nuevos con una ceremonia de apertura del semestre (Recepción a los novatos), donde los líderes hacen una presentación de la misión, de los principios y de los objetivos de la institución.

\subsection{Personas}

En relación con los criterios de compromiso con la excelencia (FNQ, 2011), el factor "personas" busca examinar la forma en que son dispuestas las condiciones para el desarrollo y utilización plena del potencial de las personas que componen la fuerza laboral, en consonancia con las estrategias organizacionales. Así, aborda los procesos gerenciales relativos a la configuración de grupos de gran desempeño, al desarrollo de las competencias de las personas y a la mantención de su bienestar.

La institución ha posibilitado un alineamiento de todos sus colaboradores, apoyándose en las evaluaciones de desempeño anuales referidas al desarrollo de personas. La estructura del plan de cargos y sueldos posibilita una progresión funcional en "Y", o sea, la progresión podrá ocurrir de manera vertical y horizontal. La práctica del Programa de Gestión por Competencias definió las evaluaciones del desarrollo de los colaboradores, con la finalidad de verificar el nivel de presencia de las competencias respectivas entre ellos. Esta estrategia es coherente con el objetivo institucional de garantizar trabajadores competentes, profesionalizados y altamente implicados con los principios de la organización.

La capacitación y el desarrollo de los docentes también son considerados en el Programa de Formación Continua, con actividades realizadas a lo largo del año, a través del Núcleo de Apoyo Pedagógico 
(NAP). En la Tabla 2 se presentan las principales acciones de capacitación realizadas durante 2013.

Tabla 2: Capacitaciones 2013

\begin{tabular}{l|c|c}
\hline Curso/conferencia & Carga horaria & No de participantes \\
\hline Formación continua para docentes & $36 \mathrm{~h}$ & 212 \\
\hline Formación continua para cuerpo técnico-administrativo & $28 \mathrm{~h}$ & 197 \\
\hline Diseminación PGQP & $10 \mathrm{~h}$ & 259 \\
\hline Capacitación interpretación de los criterios PGQP & $16 \mathrm{~h}$ & 24 \\
\hline Preparación para descripción del RG & $4 \mathrm{~h}$ & 29 \\
\hline Curso de examinador PGQP & $16 \mathrm{~h}$ & 7 \\
\hline Becas integrales latoy stricto sensu & $400 \mathrm{~h}$ & 33 \\
\hline Entrenamientos módulos sistema S.A. & $11 \mathrm{~h}$ & 215 \\
\hline Programa de desarrollo de liderazgo & $73 \mathrm{~h}$ & 98 \\
\hline
\end{tabular}

Fuente: Centro Universitario La Salle (2013).

\subsection{Procesos}

Según los criterios de compromiso con la excelencia (FNQ, 2011), el criterio "procesos" busca verificar cómo la organización reconoce los procesos para añadir valor. También identifica, gerencia, analiza y mejora los procesos principales del negocio y los procesos de apoyo, tratando separadamente los relativos a los proveedores y los económico-financieros.

Los procesos de apoyo de la institución poseen el carácter de la gestión sistémica e integrada, manteniendo indisociable la enseñanza, la investigación y la extensión, los que están en consonancia con el PDI, destacando los procesos de creación e implementación, adecuación curricular, extensión y formación académica, los que son realizados en conformidad con la legislación vigente y la demanda del mercado.

\subsection{Resultados}

De acuerdo con los criterios de compromiso con la excelencia (FNQ, 2011), el octavo y último factor se denomina "resultados" y se caracteriza por evaluar los productos de la organización, abarcando los económico-financieros y los relativos a clientes, mercados, 
sociedad, personas, procesos principales de negocios y de apoyo, así como también, los relativos a la relación con los proveedores. Este ítem se aborda considerando series históricas, las cuales están acompañadas de referenciales comparativos pertinentes para evaluar el nivel alcanzado.

A modo de síntesis es factible afirmar que en general se observan resultados objetivos de la institución a partir de un conjunto de datos cuantitativos, desplegados por cada uno de los criterios, lo que permite su correspondiente evaluación. Estos resultados se despliegan, miden, analizan y comparan con las mejores prácticas de las IES, en tres ciclos de control (2011-2013), con las siguientes categorías de resultados:

- Resultados económicos y financieros.

- Resultados relativos a los clientes y el mercado.

- Resultados relativos a la sociedad.

- Resultados relativos a las personas.

- Resultados relativos a los procesos (principal del negocio y de apoyo).

- Resultados relativos a los proveedores.

Los indicadores que se muestran en la Tabla 3 recogen los resultados del ciclo 2011-2013, los que son esenciales para evidenciar la alineación de los procesos y garantizar la calidad de la gestión, por el esfuerzo de los respectivos equipos de trabajo y el impacto generado tras la adopción de una herramienta de apoyo para la profesionalización de la gestión. 
Tabla 3: Indicadores de resultados del periodo 2011-2013

\begin{tabular}{|c|c|c|c|c|c|}
\hline & \multirow{2}{*}{\multicolumn{3}{|c|}{ Ciclos }} \\
\hline & & & & & \\
\hline 3.8 a) Resultado económicos financieros & Unid & Mejor & 2011 & 2012 & 2013 \\
\hline Gratuidades sobre los ingresos & $\%$ & $\hat{\imath}$ & 18,0 & 19,0 & 17,0 \\
\hline Compromiso de los ingresos con los gastos de personal & $\%$ & 约 & 57,8 & 62,4 & 65,0 \\
\hline Defecto sobre los ingresos (Graduación) & $\%$ & ת & 4,3 & 4,2 & 4,1 \\
\hline Efectividad de ventas (Educación Superioor Graduación) & $\%$ & $\hat{\imath}$ & 87,0 & 90,2 & 88,4 \\
\hline Ticket medio (Graduación) & $\mathrm{R} \$$ & 艺 & $2.784,47$ & $3.388,29$ & $3.418,88$ \\
\hline Crecimiento de los ingresos (Graduación) & $\%$ & $\hat{\imath}$ & 9,69 & 12,00 & 9,13 \\
\hline \multirow[t]{2}{*}{ Productos con superávit } & $\%$ & $\hat{\imath}$ & - & 97,50 & 98,00 \\
\hline & & & \multicolumn{3}{|c|}{ Ciclos } \\
\hline $3.8 \mathrm{~b})$ Resultados relativos a los clientes y el mercado & Unid & Mejor & 2011 & 2012 & 2013 \\
\hline Matrículas por semestre (Graduación) & $\mathrm{N}^{0}$ & $\hat{\imath}$ & 1966 & 2053 & 2034 \\
\hline \multirow{2}{*}{$\begin{array}{l}\text { Matrículas por inscripción para el examen de ingreso } \\
\text { Número de estudiantes por semestre (Posgrado Lato Sensu) }\end{array}$} & $\%$ & 艺 & 76,0 & 61,5 & 64,7 \\
\hline & $N^{0}$ & 艺 & 600 & 515 & 414 \\
\hline \multirow{2}{*}{$\begin{array}{l}\text { Número de estudiantes por semestre (Extensión) } \\
\text { Nivel de Internacionalización }\end{array}$} & $N^{0}$ & 艺 & 700 & 935 & 1.403 \\
\hline & $N^{0}$ & $\hat{\imath}$ & 29 & 32 & 181 \\
\hline Tasa de abandono semestral & $\%$ & ת & 14,0 & 16,3 & 14,3 \\
\hline Satisfacción del académico & $\%$ & $\hat{\imath}$ & 78,8 & 78,5 & 72,6 \\
\hline $\begin{array}{l}\text { Tasa de reclamaciones } \\
\text { Número de candidatos inareso a la universidad }\end{array}$ & $\%$ & ת & 3 & 5 & 3 \\
\hline \multirow{2}{*}{$\begin{array}{l}\text { Número de candidatos ingreso a la universidad } \\
\text { Número de candidatos por semestre }\end{array}$} & $\mathrm{N}^{0}$ & $\hat{\imath}$ & 2596 & 2829 & 3143 \\
\hline & $\%$ & $\hat{\imath}$ & 9,54 & 10,63 & 11,9 \\
\hline \multirow{3}{*}{$\begin{array}{l}\text { La retención de los estudiantes en el IES } \\
\text { Estudiantes con inscripción en programas de nivelamiento }\end{array}$} & $\mathrm{N}^{0}$ & $\hat{\imath}$ & 49 & 94 & 183 \\
\hline & $N^{0}$ & $\hat{\imath}$ & 227 & 319 & 360 \\
\hline & & \multicolumn{3}{|c|}{ Ciclos } \\
\hline $3.8 \mathrm{c}$ ) Resultados relativos a la sociedad & Unid & Mejor & 2011 & 2012 & 2013 \\
\hline $\begin{array}{l}3.8 \text { c) Resultados relativos a la sociedad } \\
\text { Proyectos y campanas sociales }\end{array}$ & $N^{0}$ & $\hat{\imath}$ & 14 & 14 & 14 \\
\hline Consumo de electricidad & $\mathrm{kw} / \mathrm{h}$ & ת & 1.943 .709 & 2.369 .202 & 2.442 .623 \\
\hline Consumo de agua & $\mathrm{m}^{3}$ & $\sqrt{2}$ & 15.552 & 24.781 & 29.635 \\
\hline Consumo de diesel & 1 & ת & 181.000 & 128.000 & 118.300 \\
\hline Estudiantes de Educación Superior en programas de prácticas & $\mathrm{N}^{0}$ & $\hat{\imath}$ & 1.896 & 1.944 & 1.871 \\
\hline $\begin{array}{l}\text { Estudiantes con el apoyo financiero para el estudio por año } \\
\text { (PROUNI) }\end{array}$ & $\mathrm{N}^{0}$ & $\hat{\imath}$ & 511,5 & 654,5 & 732 \\
\hline $\begin{array}{l}\text { Nuevos estudiantes con el apoyo financiero para el estudio por } \\
\text { año (PROUNI) }\end{array}$ & $\mathrm{N}^{0}$ & $\hat{\imath}$ & 251 & 202 & 193 \\
\hline Tendimientos en Clínica Integrado de Salud (CIS) & $N^{0}$ & $\hat{\imath}$ & 10375 & 8815 & 12714 \\
\hline $\begin{array}{l}\text { Atendimiento a la comunidad en Núcleo de Prácticas Jurídicas } \\
\text { (NPJ) }\end{array}$ & $N^{0}$ & $\hat{\imath}$ & 600 & 580 & 580 \\
\hline Personas beneficiarios de la Pastoral & $\mathrm{N}^{0}$ & $\hat{\imath}$ & 3700 & 3540 & 5200 \\
\hline & & & & Ciclos & \\
\hline $3.8 \mathrm{~d}$ ) Resultados relativos a las personas & Unid & Mejor & 2011 & 2012 & 2013 \\
\hline Satisfacción de los empleados & $\%$ & 仓 & 69,6 & NA & 67,2 \\
\hline Horas de formación por empleados & h & 仓े & 98,9 & 112,5 & 156,36 \\
\hline Horas extraordinarias por horas trabajadas & $\%$ & $\sqrt{2}$ & 1,5 & 1,9 & 3,6 \\
\hline Ausentismo & $\%$ & 约 & 1,8 & 1,4 & 2,27 \\
\hline Rotación & $\%$ & $\sqrt{3}$ & 2,5 & 2,0 & 1,87 \\
\hline Educación de la fuerza de trabajo (nivel superior) & $\%$ & $\hat{\imath}$ & 52,0 & 61,7 & 59,1 \\
\hline
\end{tabular}



CALIDAD Y PRODUCTIVIDAD: EL CASO DE UNILASALLE DE BRASIL - P. Fossatti, R. Vieira de Souza y F. Ganga

\begin{tabular}{|c|c|c|c|c|c|}
\hline & & & \multicolumn{3}{|c|}{ Ciclos } \\
\hline 3.8 e) Resultados relativos a procesos principales y de apoyo & Unid & Mejor & 2011 & 2012 & 2013 \\
\hline Índice de Redcompra (Graduación y Post-graduación) & $\%$ & 仓े & 1,78 & 2,33 & 2,42 \\
\hline Índice General del Curso - IGC & Nota & 仓 & 3 & 3 & 3 \\
\hline Procedimientos normalizados de trabajo & $\%$ & 艺 & 8 & 14 & 30 \\
\hline $\begin{array}{l}\text { Cursos que participan en proyectos Tecnosocial Igraduación y } \\
\text { Posgraduación) }\end{array}$ & $\%$ & $\hat{\imath}$ & 24,5 & 35,3 & 30,6 \\
\hline Préstamo de materiales de la biblioteca & $\mathrm{N}^{0}$ & 仓 & 82.377 & 65.427 & 62.985 \\
\hline Asistencia a la publicación de revistas científicas & $\%$ & 出 & - & 81 & 72 \\
\hline Renovación de materiales de la biblioteca & $\mathrm{N}^{0}$ & 仓 & 100.500 & 89.489 & 88.470 \\
\hline Tratamiento en soporte, redes y sistemas & $\mathrm{N}^{0}$ & 仓 & 2.964 & 3.326 & 4.385 \\
\hline & & & \multicolumn{3}{|c|}{ Ciclos } \\
\hline $3.8 \mathrm{ff}$ Resultados relativos a los proveedores & Unid & Mejor & 2011 & 2012 & 2013 \\
\hline Indice de rendimiento globales de proveedores & $\%$ & $\hat{\imath}$ & - & - & 70,0 \\
\hline Economía en el proceso de compra & $\%$ & 仓े & 1,41 & 1,36 & 1,24 \\
\hline Compras con entrega en el plazo de entrega & $\%$ & $\hat{\imath}$ & 93,7 & 94,8 & 78,4 \\
\hline
\end{tabular}

Fuente: Centro Universitario La Salle (2013).

Como ha quedado de manifiesto, el trabajo ha intentado presentar de forma práctica los criterios de gestión que identifican, controlan y buscan el compromiso con las metas en cada uno de los respectivos indicadores. Se ha pasado revista a la alineación de las estrategias, la búsqueda de la eficacia de la gestión y el compromiso con la excelencia.

\section{Conclusiones}

El artículo ha tratado de analizar la contribución del PGQP como herramienta para la profesionalización de la administración de la Unilasalle Canoas. El estudio de caso tipo cualitativo con análisis documental ha resultado en discursos y prácticas emergentes de los criterios del PGQP que colaboran con el mejoramiento del modelo de gestión universitaria de la Unilasalle.

La Unilasalle se caracteriza por atender a los principios de una IES comunitaria, filantrópica y confesional, anclada en herramientas de gestión como BSC y PGQP. La opción por la herramienta PGQP presenta resultados significativos en todos sus criterios analizados, también en el diálogo con los principios lasallistas. 
Fue factible evidenciar un cambio de cultura organizacional en sus discursos y prácticas en la gestión entre las que se puede mencionar el nuevo vocabulario, nuevos conocimientos teóricos, uso adecuado de la información, visión sistémica de la IES, formación continuada y surgimiento de un liderazgo gestor. En este sentido, por ejemplo, se percibe que las personas están utilizando nueva terminología en la organización, añadiendo en su comunicación cotidiana conceptos como: BSC, PGQP, PDCA, 5W2H, indicadores, metas, mapas estratégicos, alineación de las acciones, resultados.

Los nuevos conocimientos teóricos provenientes del PGQP han permitido la optimización de los recursos institucionales.

El saber acerca de la gestión por resultados a partir de indicadores y metas es otro conocimiento resultante de los datos como factores de crecimiento.

El uso adecuado de la información estratégica revela el surgimiento de nuevas prácticas, realidad que comienza a tornarse institucional.

La visión sistémica de la IES se muestra también en el desplazamiento de la micro a la macro óptica institucional, facilitando la aproximación hacia un diálogo de las áreas académicas y administrativas.

La formación continua también es producto de una inquietud institucional, lo que queda en evidencia a través de innumerables actividades donde los participantes del BSC y del PGQP se encuentran embarcados. Estas acciones formativas tienen relación con las nuevas estrategias del mercado y las políticas de formación continua, materializadas en cursos regulares, conferencias, congresos, cursos de corta duración y acceso a los medios de comunicación.

El surgimiento de un liderazgo gestor tiene su punto de partida en una práctica basada en el trabajo en equipo, en la pedagogía de proyectos y en el foco en los indicadores, metas y resultados. Este nuevo modo de percibir y de demostrar consecuencias de la gerencia institucional se refleja en la promoción genuina del desarrollo de 
184 PROFESIONALIZACIÓN DE LA GESTIÓN DESDE EL PROGRAMA GAUCHO DE CALIDAD Y PRODUCTIVIDAD: EL CASO DE UNILASALLE DE BRASIL - P. Fossatti, R. Vieira de Souza y F. Ganga

personas, en el aumento del profesionalismo, en el compromiso laboral y en la satisfacción con el trabajo que está siendo realizado.

También es digno de destacar que se observó de manera nítida, que los resultados presentados en el Informe de Gestión sobre el PGQP están alineados con la misión y visión de la IES, comprobando con ello que existe un despliegue de objetivos y metas a partir del uso del BSC, traducido en el respectivo mapa estratégico de la institución.

Finalmente, y considerando el proceso de envío de información al sistema de evaluación (donde los resultados representan el 40\% de la calificación), se debe resaltar que estos fueron esenciales para medir y presentar el desempeño de la IES en el ciclo de análisis 20112013. En este sentido, se observó que la mayoría de los indicadores muestran una evolución positiva, aunque aquellos que no presentaron esa evolución fueron redireccionados a partir de las justificaciones presentadas y los planes de acción desplegados, de tal forma de apoyar a la IES en el proceso de mejora continua de la gestión en el próximo ciclo.

A partir de lo sistematizado, es indudable que se abren nuevas rutas investigativas, las cuales debieran considerar los análisis comparados con otras universidades lasallistas, con instituciones públicas y privadas $\mathrm{y}$, a futuro, intentar estudios que abarquen toda Iberoamérica.

\section{Referencias}

Bardin, L. (2011). Análise de conteúdo. São Paulo: Edições 70.

Betancourt, J. (2002). Gestión estratégica: navegando hacia el cuarto paradigma. Venezuela: T.G. Red 2000 Ediciones. Recuperado de http://www. eumed.net/libros-gratis/2006c/220/

Braga, R., \& Monteiro, C. A. (2005). Planejamento estratégico sistêmico para instituições de ensino. São Paulo: Hoper.

Presidência da República. (2013). Instituições comunitárias de educação superior. LEI No 12.881/2013 (Lei Ordinária) del 12/11/2013. Recuperado de http://www.planalto.gov.br/ccivil_03/_Ato2011-2014/2013/Lei/ L12881.htm 
Bustos, J., Zapata, M. y Ramírez, M. (2008). Más allá de la gestión estratégica en educación superior: aplicación del cuadro de mando integral. Oikos: Revista de la Escuela de Administración y Economía, 26, 95-144.

Cauchick Miguel, P.A. (2004). Princípios de gestão baseada no Prêmio Nacional da Qualidade. En E.S. Siqueira \& V.R.E. Spers, Gestão e negócios, entre o social e o administrativo pp. 210- 234. São Paulo: Edições O.L.M.

Centro Universitário La Salle. (2013). Relatório de gestão Programa Gaúcho da Qualidade e Produtividade. Canoas: Brasil.

Cortadellas, J. (2007). Dirección estratégica y calidad total ¿también en las universidades? En CINDA y Cátedra Unesco de Dirección Universitaria (CUDU), Acreditación y dirección estratégica para la calidad de las universidades, pp. 259-271. Santiago de Chile.

De la Orden, A., Asensio, I., Carballo, R., Fernández Díaz, J., Fuentes, A., García Ramos, J.M. y Guardia, S. (1997). Desarrollo y validación de un modelo de calidad universitaria como base para su evaluación. RELIEVE, 3, 1. Recuperado de http://www.uv.es/RELIEVE/v3nl/ RELIEVEv3n1_2.htm

De la Orden, A. (2012). Innovación, evaluación y calidad en la educación. Revista de Evaluación Educativa, 1 (1), 1-21. Recuperado de: http:// revalue.mx/revista/index.php/revalue/issue/view/2

Drucker, P. F. (2009). O melhor de Peter Drucker: a administração. São Paulo: Nobel.

Fundação Nacional da Qualidade. (2011). Critério compromisso com a excelência - avaliação e diagnóstico da gestão organizacional. São Paulo: FNQ. Recuperado de http://www.mbc.org.br/mbc/pgqp/hot_sites/ premio2014/arquivos///compromisso_vsPDF_PGQP.pdf

Fossatti, P., Hengemüle, E. \& Casagrande, C. A. (Org). (2011). Ensinar a bem viver. Canoas: Ed. UnilaSalle.

Fox, D. (1981). El proceso de investigación en educación. Pamplona: Eunsa.

Ganga, F. y Burotto, J. (2012). Asimetrías de información entre agente y principal de las universidades chilenas. Revista de Estúdios Gerenciales 28 (122), 83-104.

Ganga, F., Cassinelli, A., Piñones, M., y Quiroz, J. (2014). El concepto de eficiencia organizativa: una aproximación a lo universitario. Revista Lider, 25, 126-150.

Ganga, F., Ramos, E., Leal, A., y Pérez, K. (2015). Administración estrastégica: Aplicación del cuadro de mando integral (CMI) a uma organización no gubernamental. Revista Ciencias Sociales (RCS), XXI, 1, 136-159. 
International Association La Salle Universities. (2013). Members. Recuperado de http://www.lasalle-ialu.org/list_institutions?region=5

Movimento Brasil Competitivo. (2012). PGQP - Qualidade RS. Porto Alegre. Recuperado de http://www.mbc.org.br/mbc/pgqp/

Movimento Brasil Competitivo. (2014). PGQP - Qualidade RS. Porto Alegre. Recuperado de http://www.mbc.org.br/mbc/pgqp/

Rodríguez, S. (1991). Calidad universitaria: un enfoque institucional y multidimensional. En, La evaluación de las instituciones universitarias (pp. 39-72). Editores: Ministerio de Educación Cultura y Deporte, Consejo de Universidades. España.

Senge, P. (1992). La quinta disciplina. Barcelona: Granica.

Yáñez, J. (2012). Nuevo enfoque acerca de la calidad de la educación. Revista Docencia, 46, 108-111.

Zúñiga, M. (2007). Algunas consideraciones sobre el aseguramiento de la calidad. En CINDA y Cátedra Unesco de Dirección Universitaria (CUDU). Acreditación y dirección estratégica para la calidad de las universidades (pp. 71-80). Santiago de Chile.

Recibido: 31/12/2014

Aceptado: 07/05/2015 